\title{
Potential Therapeutic Effects of Lipoic Acid on Memory Deficits Related to Aging and Neurodegeneration
}

\author{
Patrícia Molz ${ }^{1,2}$ and Nadja Schröder ${ }^{1,2 *}$ \\ ${ }^{1}$ Graduate Program in Medicine and Health Sciences, Faculty of Medicine, Pontifical Catholic University, Porto Alegre, Brazil, \\ ${ }^{2}$ Neurobiology and Developmental Biology Laboratory, Faculty of Biosciences, Pontifical Catholic University, Porto Alegre, \\ Brazil
}

The aging process comprises a series of organic alterations, affecting multiple systems, including the nervous system. Aging has been considered the main risk factor for the advance of neurodegenerative diseases, many of which are accompanied by cognitive impairment. Aged individuals show cognitive decline, which has been associated with oxidative stress, as well as mitochondrial, and consequently energetic failure. Lipoic acid $(\llcorner A)$, a natural compound present in food and used as a dietary supplement, has been considered a promising agent for the treatment and/or prevention of neurodegenerative disorders. In spite of a number of preclinical studies showing beneficial effects of LA in

OPEN ACCESS

Edited by:

Antonella Gasbarri,

University of L'Aquila, Italy

Reviewed by:

Enrique Cadenas,

University of Southern California,

United States

Cedric Williams,

University of Virginia, United States

*Correspondence:

Nadja Schröder

nadja.schroder@pucrs.br

Specialty section:

This article was submitted to

Neuropharmacology,

a section of the journal

Frontiers in Pharmacology

Received: 26 June 2017 Accepted: 06 November 2017 Published: 12 December 2017

Citation:

Molz P and Schröder N (2017) Potential Therapeutic Effects of Lipoic Acid on Memory Deficits Related to Aging and Neurodegeneration.

Front. Pharmacol. 8:849. doi: 10.3389/fphar.2017.00849 memory functioning, and pointing to its neuroprotective potential effect, to date only a few studies have examined its effects in humans. Investigations performed in animal models of memory loss associated to aging and neurodegenerative disorders have shown that LA improves memory in a variety of behavioral paradigms. Moreover, cell and molecular mechanisms underlying LA effects have also been investigated. Accordingly, LA displays antioxidant, antiapoptotic, and anti-inflammatory properties in both in vivo and in vitro studies. In addition, it has been shown that LA reverses age-associated loss of neurotransmitters and their receptors, which can underlie its effects on cognitive functions. The present review article aimed at summarizing and discussing the main studies investigating the effects of $L A$ on cognition as well as its cell and molecular effects, in order to improve the understanding of the therapeutic potential of LA on memory loss during aging and in patients suffering from neurodegenerative disorders, supporting the development of clinical trials with $L A$.

\section{Keywords: memory, lipoic acid, neuroprotection, aging, neurodegenerative disorders}

\section{INTRODUCTION}

Aging is a multifactorial process that involves genetics, lifestyle, and environmental factors (Hagen et al., 1999; Savitha and Panneerselvam, 2006; Lopez-Otin et al., 2013; Kennedy et al., 2014). During aging, biological processes promote the gradual loss of the individual's ability to maintain homeostasis, followed by a progressive deterioration in biochemical and physiological functions of the organism, increasing the susceptibility to diseases associated with aging (Arivazhagan et al., 2001a; Kumaran et al., 2005; Singh et al., 2015). Cognitive function also declines with age (Liu, 2008). 
Aging has been associated to a more oxidized state in the redox balance (Jones and Sies, 2015), and the central nervous system becomes vulnerable to oxidative stress (Arivazhagan et al., 2002; Kidd, 2005; Ferreira et al., 2013; Zuo and Motherwell, 2013), defined as an unbalanced redox signaling, related to increased amounts of oxidants and ineffective antioxidant defenses (Go and Jones, 2017). In this context, nutrition can be considered a critical life-style factor that impacts the development and progression of neurodegenerative diseases (Virmani et al., 2013). Dietary supplementation with mitochondrial nutrients could promote natural neuroprotective effects, delaying the onset or progression of cognitive dysfunction and neurodegenerative diseases (Miquel, 2002; Abadi et al., 2013; Di Domenico et al., 2015; Mehrotra et al., 2015).

Over the years, lipoic acid (LA) has received increased attention as a nutritional supplement with therapeutic potential in the treatment or prevention of different pathologies (Shay et al., 2009; Rochette et al., 2013; Park et al., 2014), such as neurodegenerative diseases (Packer et al., 1997; Hager et al., 2001; Holmquist et al., 2007; Farr et al., 2012). LA has been shown to improve mitochondrial function (Kidd, 2005; Zhang et al., 2010; Zuo and Motherwell, 2013; Hiller et al., 2016), in addition to protect from cognitive dysfunction associated to aging and neurodegenerative diseases (Hager et al., 2007; Moreira et al., 2007; Liu, 2008). Thus, this study aims to review and discuss main findings showing potential memory-improving effects, as well as neuroprotective effects of LA, giving support to its use as an adjuvant in the treatment of neurodegenerative disorders. For this, we focused in discussing experimental studies addressing behavioral evaluations and cellular and molecular effects of LA.

\section{LIPOIC ACID}

LA (1,2-dithiolane-3-pentonoico acid, thioctic acid) (Shay et al., 2009) was discovered in 1937 by Snell et al. (1937) and characterized by Reed et al. (1951). LA has been considered a powerful micronutrient presenting a range of pharmacological properties (Rochette et al., 2013; Koufaki, 2014; Park et al., 2014); however, many aspects of LA effects still need to be clarified, especially its application in the treatment and prevention of neurodegeneration.

\section{Chemistry of LA}

LA is a low molecular weight dithiol with a chiral center containing eight-carbon disulfide in its structure (Figure 1; De Araujo et al., 2011). It is naturally occurring in all prokaryotic and eukaryotic cells (Bast and Haenen, 2003; Park et al., 2014). LA acts as co-factor in multienzyme complexes in the mitochondria, such as pyruvate dehydrogenase and $\alpha$-ketoglutarate dehydrogenase (Holmquist et al., 2007; Ghibu et al., 2009). A substantial part of LA is reduced to dihydrolipoic acid (DHLA) by lipoamide dehydrogenase (E3 component of the pyruvate dehydrogenase complex and $\alpha$-ketoglutarate dehydrogenase) with involvement of the NADH and NADPH system (Arivazhagan et al., 2001b; Bilska et al., 2007). Reduction of LA to DHLA may also be completed by other cellular reducing systems, including

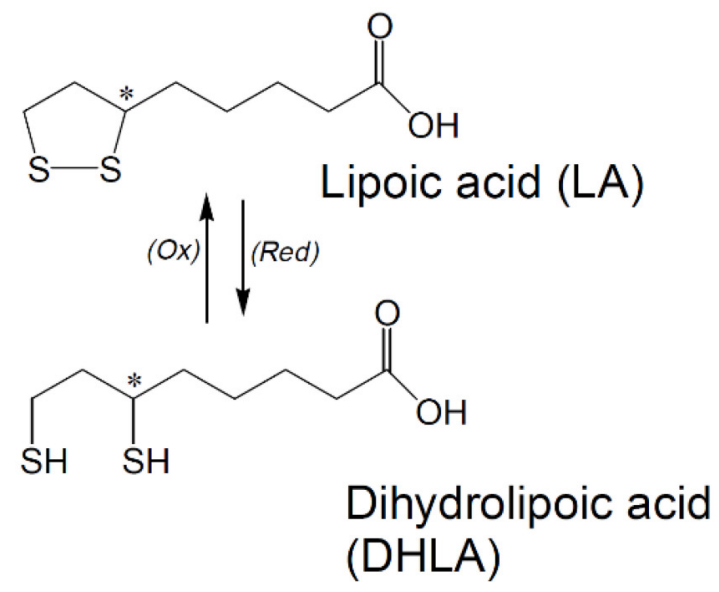

FIGURE 1 | Chemical structure of lipoic acid $(L A)$ and dihydrolipoic acid (DHLA). *Indicates a chiral Carbon atom.

$\mathrm{NAD}(\mathrm{P}) \mathrm{H}$-driven enzymes, such as thioredoxin reductases (Rochette et al., 2013). LA also contains an asymmetric carbon resulting in two optical isomers, the $\mathrm{S}$ form and the $\mathrm{R}$ form, with the former being synthesized endogenously (De Araujo et al., 2011).

\section{Dietary Sources, Metabolism, Toxicity, and Nutritional Recommendations}

LA may be obtained from the diet (Packer et al., 1997; Hagen et al., 1999; Morikawa et al., 2001; Ghibu et al., 2009) and due to its capability of endogenous synthesis it is not considered a vitamin (Packer et al., 1995, 2001; Bast and Haenen, 2003), but is structurally considered a member of the vitamin B family (Xing et al., 2015). When ingested as a nutritional supplement preferably in the racemic mixture form, LA contains two isomers (1:1 of R-LA and S-LA), in which S-LA may prevent polymerization of R-LA and thus increases the bioavailability of the later (De Araujo et al., 2011).

LA is found in both vegetable and animal-based foods, identified as lipoyl-lysine (coupling of LA to specific lysine residues; Ghibu et al., 2009; Shay et al., 2009; Rochette et al., 2013; Li et al., 2015). R-LA is most abundantly found in vegetables such as spinach, broccolis, and tomatoes, which contain, respectively, $3.15 \pm 1.11,0.94 \pm 0.25$, and $0.56 \pm 0.23 \times 10^{-3} \mathrm{~g}$ lipoyl-lysine (gram dry weight). In foods of animal origin, the highest amounts of lipoyl-lysine are found in bovine kidney, heart, and liver, containing, respectively, $2.64 \pm 1.23,1.51 \pm 0.75$, and $0.86 \pm$ $0.33 \times 10^{-3}$ g lipoyl-lysine (gram dry weight) (Lodge and Packer, 1999).

The biochemistry and pharmacokinetics of LA have been extensively reviewed elsewhere (Packer et al., 1995; Bustamante et al., 1998; Cremer et al., 2006b; Shay et al., 2009; Rochette et al., 2013). In summary, either from food or nutritional supplement sources, LA is rapidly absorbed, metabolized and excreted (Moini et al., 2002; Rochette et al., 2013). LA is absorbed rapidly from the gastrointestinal tract (Hagen et al., 1999; Jalali-Nadoushan 
and Roghani, 2013), up to $93 \%$ of a dose administered orally is absorbed in the gastrointestinal tract, (Cremer et al., 2006b) and is clearly subject to considerable pre-systemic elimination (Hagen et al., 1999). Approximately $27-34 \%$ LA orally administered is available for absorption by the tissues (Hagen et al., 1999) and the liver is one of the main clearance organs because it has a high absorption and storage capacity (Bustamante et al., 1998; Hagen et al., 1999).

Studies have shown that the gastrointestinal absorption of LA is highly variable and its efficiency appears to be reduced when ingested in the diet, suggesting that the absorption of LA competes with other nutrients (Packer et al., 2001; Shay et al., 2009; Rochette et al., 2013). Gastrointestinal uptake of LA is fast and its presence in the plasma is followed by a fast clearance (Shay et al., 2009). The plasmatic half-life of LA is $30 \mathrm{~min}$. Endogenous plasma levels of LA and DHLA in humans are, respectively, $1-25 \times 10^{-9} \mathrm{~g} / \mathrm{mL}$ and $30-140 \times 10^{-9} \mathrm{~g} / \mathrm{mL}$ (Ghibu et al., 2009; Rochette et al., 2013). Urinary excretion is maximal $3-6 \mathrm{~h}$ following LA administration. Approximately $45 \%$ is excreted in the urine during the first $24 \mathrm{~h}$ and only $3 \%$ is excreted in the feces. Moreover, only a small amount of the administered LA is excreted in the unaltered form (Bustamante et al., 1998).

There are no recommendations for daily LA intake in humans. However, the safety of LA was determined in acute and subchronic toxicity studies as well as its mutagenicity/genotoxicity in in vitro and in vivo studies. In dogs, a $\mathrm{LD}_{50}$ of $400-500 \mathrm{mg} \mathrm{LA} / \mathrm{kg}$ b.w. has been reported (Packer et al., 1995). In rats a $\mathrm{LD}_{50}$ of $2,000 \mathrm{mg} / \mathrm{kg}$ b.w. was described, with some rats presenting signs of reduced well-being, including sedation, apathy, piloerection, hunched posture, and/or eye closure (Cremer et al., 2006b). On the other hand, studies evaluating oral LA supplementation up to $60 \mathrm{mg} / \mathrm{kg}$ per day in rats showed no adverse effects concerning body weight, histopathological findings, and blood analyses (Cremer et al, 2006a,b). Therefore a NOAEL (no observed adverse effect level) for rats of $60 \mathrm{mg} / \mathrm{kg} /$ day for longterm LA supplementation was provided. Clinical trials using LA to assess adverse health effects in humans were performed in doses up to $2,400 \mathrm{mg} /$ day with no reported adverse effects vs. placebo (Shay et al., 2009). In spite of that, the exact doses that could induce adverse human health effect are still to be set up.

\section{NEURODEGENERATIVE DISORDERS}

Neurodegenerative diseases are a heterogeneous group of disorders described by progressive and selective neuronal death with degeneration of specific brain regions (Arivazhagan and Panneerselvam, 2002; Lin and Beal, 2006; Savitha and Panneerselvam, 2006), often associated with abnormal deposits of proteins in neurons or extracellularly (Chen et al., 2012). Neurodegeneration is characterized by its insidious and chronic progressive onset and aging has been considered the main risk factor (Lin and Beal, 2006; Chen et al., 2012; Virmani et al., 2013; Irwin et al., 2016).

During aging, deleterious changes accumulate, causing the gradual decline of the biochemical and physiological functions
(Arivazhagan and Panneerselvam, 2002; Kumaran et al., 2005; Santos et al., 2013). Moreover, aging individuals are susceptible to degeneration of selective brain regions (Aliev et al., 2009), increasing the incidence of diseases such as Alzheimer's, Parkinson's, Huntington's diseases, among others (Lin and Beal, 2006; Bagh et al., 2011; Irwin et al., 2016).

Over the last decades, a wide range of studies have shown that progression of neurodegeneration is associated with increased DNA damage (partly is attributed to an imbalance between antioxidant and prooxidant factors; Chen et al., 2012; Kim et al., 2015). In addition, mitochondrial decline, leading to cognitive dysfunction (Liu, 2008; Aliev et al., 2009; Bishop et al., 2010; Irwin et al., 2016) as well as aggregation of oxidized proteins, accumulation of metals, inflammation and excitotoxicity have been reported (Moreira et al., 2007). Oxidative stress and mitochondrial dysfunction are interrelated mechanisms that play a central role in aging brain (Santos et al., 2013), since the continuous generation of reactive oxygen species (ROS), mainly the superoxide anion $\left(\mathrm{O}_{2}^{-\bullet}\right)$ at complexes I and III of the mitochondrial respiratory chain, throughout life produces a sustained oxidative stress by aging, possibly resulting in cognitive impairments (Limoli et al., 2004; Kumaran et al., 2005; Bagh et al., 2011). Although the exact mechanisms underlying the effects of unbalanced redox signaling are not completely elucidated, studies suggest that it plays a role in the pathogenesis of neurodegenerative disorders (Liu et al., 2017).

Brain aging has been related to structural alterations and inflammation, accompanied by cognitive and memory dysfunctions (Bagh et al., 2011; Pizza et al., 2011; Thakurta et al., 2014). With the aging of the world population and the increasing life expectancy, the risk of developing neurodegenerative diseases is higher than ever, as they are affecting millions of people each year in epidemic proportions (Santos et al., 2013; Irwin et al., 2016). As a result, an inevitable socioeconomic burden on our health care systems will occur. Effective prophylactic and therapeutic treatments are urgent for this group of seemingly inexorable diseases. In this review we will focus on discussing the ameliorating effects of LA on cognitive deficits observed in animal models of aging and neurodegenerative disorders.

\section{EFFECTS OF LA IN EXPERIMENTAL MODELS OF MEMORY DEFICITS ASSOCIATED TO NEURODEGENERATIVE DISORDERS}

\section{Behavioral Studies}

Alzheimer's Disease

Alzheimer's disease (AD) is the most common neurodegenerative disorder that causes dementia and affects middle to old-aged individuals (Hager et al., 2001; Gonzalez et al., 2014; Irwin et al., 2016). AD is characterized by progressive loss of cognitive functions, including memory, language, and reasoning (Di Domenico et al., 2015).

Studies have investigated the effects of LA in experimental AD models (Jesudason et al., 2005; Siedlak et al., 2009; Ahmed, 2012; 
Sancheti et al., 2013). For instance, Quinn et al. (2007) evaluated the chronic dietary supplementation with LA on hippocampusdependent memory of aged Tg2576 mice, a transgenic model of cerebral amyloidosis associated with AD. LA treatment was shown to reduce hippocampal-dependent memory deficits, significantly improving learning and memory in the Morris water maze in comparison to Tg2576 mice that did not receive LA. However, no significant differences in $\beta$-amyloid levels were found between $\operatorname{Tg} 2576$ mice that received LA in comparison to the ones that did not receive LA, indicating that chronic LA supplementation in the diet can ameliorate hippocampal memory impairments in Tg2576 mice without any effect on $\beta$-amyloid levels or plaque deposition.

Another study assessed the effects of LA in senescenceaccelerated mouse prone 8 (SAMP8) mice, associated to learning and memory impairments, and showed that LA can improve memory, in different paradigms (Farr et al., 2012). In object recognition, results indicated that mice that received LA presented a higher memory index than vehicle-treated mice. When memory was tested in the Barnes maze, results indicated that LA-treated mice spent more time by the target, and made fewer errors than controls, but did not present differences in speed in traversing the maze (distance/time) during training.

On the other hand, Siedlak et al. (2009) investigated young and aged mice overexpressing amyloid- $\beta$ precursor protein (APP) and controls and showed that administration of R-LA had little effect on Y-maze performance. The authors concluded that, although oxidative stress has been proposed to mediate amyloid pathology and cognitive decline in aging, long-term LA administered within tolerable nutritional levels, presented limited benefit.

\section{Parkinson's Disease}

Parkinson's disease (PD) is the second most frequent neurodegenerative disorder in aging individuals and features motor symptoms related to dopaminergic neuronal loss in the substantia nigra, which results in decreased striatal dopaminergic terminals (Beal, 2003; De Araujo et al., 2011). Studies have shown that, in addition to rescuing cognitive deficits, LA is also able to ameliorate motor impairment related to PD. The effects of LA were examined in a rat model of PD induced by rotenone. The effect of LA $(50 \mathrm{mg} / \mathrm{kg} / \mathrm{day}$, p.o.) was evaluated after the administration of rotenone in the open-field and square bridge tests. The authors reported that LA improved rotenone-induced behavioral deficits. In the open-field test, LA significantly increased the ambulation frequency, increased the number of stops, elevated the activity index and lessened the inactive sittings, but did not increase the rearing frequency in comparison to the group that received rotenone. In the Square bridge test, treatment with LA protected the rats from falling as compared to rotenone group (Zaitone et al., 2012).

Jalali-Nadoushan and Roghani (2013) investigated the effect of LA (at doses of 50 and $100 \mathrm{mg} / \mathrm{kg}$ ) in a 6-hydroxydopamine (6-OHDA)-induced model of hemi-parkinsonism and observed significantly attenuated rotations on behavioral testing, induced by both doses.
The effects of LA in lipolysaccharide (LPS)-induced inflammatory PD model were also evaluated (Li et al., 2015), and the results showed that LA treatment $(100 \mathrm{mg} / \mathrm{kg} / \mathrm{d})$ partially improved motor dysfunction. No significant recovery was observed in dyskinesia in PD mice that received LA. However, a significant amelioration was observed in the adhesive removal test, in which LA treatment significantly decreased the reaction time in comparison to the LPS group.

\section{Huntington's Disease}

Huntington's disease (HD) is a chronic neurodegenerative disease and a hereditary autosomal-dominant disorder of the central nervous system caused by a single genetic mutation (Ross et al., 2014), characterized by neuronal death in caudate and putamen and in the cerebral cortex, and to a lesser extent in hippocampus and subthalamic nucleus (Mehrotra et al., 2015). This disorder is classically characterized by motor symptoms and cognitive and behavioral features (Ross et al., 2014). A HD model that has been easily replicated in animals is based on the treatment with 3-nitropropionic acid (3-NP), which promotes development of mitochondrial dysfunctions leading to bioenergetic failure (energy impairment, oxidative stress, and excitotoxicity). A study by Mehrotra et al. (2015) investigated the effects of LA in 3-NP-induced HD in rats. Administration of LA improved spatial memory acquisition and retrieval assessed using the Morris water maze. Analysis of time taken and distance traveled to find the platform in the target quadrant revealed that LA supplementation for 21 days to 3-NP-treated animals resulted in a lower latency and the distance traveled was also reduced. In addition, the average number of platform crossings in the probe trial was increased in 3-NP treated animals that received LA. Thus, the authors demonstrated that LA supplementation improved spatial memory by ameliorating the iron- and copperinduced oxidative injury observed in age-related disorders. In the Y-maze test, animals display a preference to explore the novel arm of the maze, making fewer entrances in the previously explored arm, due to spontaneous alternation. The authors showed that 3-NP-treated animals traveled a significantly lower distance, assessed by the number of entries in the novel arm, and that supplementation with LA reversed these deficits.

\section{Aging Models}

Consistent evidence indicate that memory is affected by aging in rodents as well as in humans. A study by Liu et al. (2002a) investigated the effects of LA supplementation $(0.1 \%$ in the diet $)$ on spatial memory tested in the Morris water maze, and temporal memory using the peak procedure (time-discrimination procedure) in old rats. Results showed that LA supplementation alone or combined with another mitochondrial metabolite, acetyl-1-carnitine, improved both spatial and temporal memory. In 12-month old SAMP-8 mice, chronic LA administration improved cognition in both the T-maze footshock avoidance paradigm and the lever press appetitive task without inducing non-specific locomotor effects (Farr et al., 2003).

LA has also been reported to improve behavior of aged mice in an open-field memory test (Stoll et al., 1993, 1994), in a Morris 
water maze test (Stoll et al., 1994; Liu et al., 2002a). LA also and ameliorated acquisition and retrieval in a dose-dependent manner, in old female NMRI mice, in the active avoidance learning test (Stoll et al., 1994).

\section{Other Models of Neurotoxicity}

Cui et al. (2006) evaluating a concomitant treatment with LA and d-Galactose exposure (used to induce memory loss and neurodegeneration) verified that LA ameliorated memory dysfunction in the Morris water maze task. Another study reported the neuroprotective effects of LA in neurotoxicity model induced by $\mathrm{AlCl}_{3}$ administration to mice (Mahboob et al., 2016). LA enhanced fear memory and social novelty preference in comparison to the $\mathrm{AlCl}_{3}$-treated group.

In summary, current evidence indicates that LA is able to improve memory, reversing impairments associated to a variety of experimental models of neurodegenerative disorders, and exposure to neurotoxicants, as well as normal aging. Table 1 summarizes in vivo studies investigating the neuroprotective effects of LA on behavioral parameters. Furthermore, LA was also shown to act as a memory-improving molecule in different learning and memory paradigms, including aversive, spatial, and recognition memory.

LA has been tested in humans, in studies by Hager and coworkers (Hager et al., 2001, 2007), as a treatment option for $\mathrm{AD}$. The authors examined the effect of LA for 24 and 48 months and observed that the treatment lead to a stabilization of cognitive function, verified by unchangeable records in two neuropsychological tests, mini-mental state examination (MMSE) and the $\mathrm{AD}$ assessment score, cognitive subscale (ADAScog).

\section{PUTATIVE MECHANISMS UNDERLYING LA-INDUCED NEUROPROTECTIVE EFFECTS}

In vivo as well as in vitro studies have been performed in order to characterize cellular and molecular effects of LA underlying its memory-ameliorating activities (Table 2). The effects of LA on oxidative markers in various brain regions have been discussed in different studies in animals models of aging and neurodegenerative diseases (Cui et al., 2006; Ferreira et al., 2009; Militao et al., 2010; Farr et al., 2012). LA administration decreases lipid peroxidation evaluated by MDA (Arivazhagan and Panneerselvam, 2000; Arivazhagan et al., 2002; Liu et al., 2002b; Ferreira et al., 2009; Militao et al., 2010; Farr et al., 2012) in different brain regions, and elevates the activities of antioxidants such as ascorbate (vitamin C), $\alpha$ tocoferol (vitamin E) (Arivazhagan and Panneerselvam, 2000), glutathione (GSH) (Arivazhagan and Panneerselvam, 2000; Farr et al., 2012), superoxide dismutase (SOD) activity (Arivazhagan et al., 2002; Cui et al., 2006; Militao et al., 2010), catalase (CAT) (Arivazhagan et al., 2002; Militao et al., 2010), glutathione peroxidase (GSH-Px) (Arivazhagan et al., 2002; Militao et al., 2010), glutathione redutase (GR) (Arivazhagan et al., 2002), glucose-6-P-dehydrogenase (G6PDH) (Arivazhagan et al., 2002).
Moreover, administration of LA reversed the augmentation of protein carbonyls levels in a radiation-induced cognitive dysfunction model (Manda et al., 2007), and decreased the protein carbonyls levels in aged SAMP8 mice (Farr et al., 2003).

A study by Zaitone et al. (2012) showed that LA increased striatal dopamine levels and significantly increased GSH and CAT activity in the striatum in a PD experimental model. In reserpine-treated rats, LA enhanced the amount of GSH, while diminishing GSSG levels in the striatum. Moreover, LA decreased NO concentrations in striatum and pre-frontal cortex, without significantly affecting S-nitrosothiol levels. LA also increased enzymatic activities of GPx and GST in the striatum (Bilska et al., 2007). Reserpine significantly decreased enzymatic activity of L$\gamma$-glutamyl transpeptidase $(\gamma$-GT), while pretreatment with LA was able to restore it.

The effects of LA on oxidative stress in rotenone parkinsonian rat brains were investigated, showing that LA can reduce lipid peroxidation and protein carbonylation (Zaitone et al., 2012). LA also lowered the levels of MDA and nitrite in the 6-OHDA-induced rat model of hemi-parkinsonism (JalaliNadoushan and Roghani, 2013). Karunakaran et al. (2007) analyzed the protective effect of LA in the MPTP mouse model of $\mathrm{PD}$, demonstrating that coadministration with LA prevents the activation of apoptosis signal regulating kinase (ASK1) signaling cascade and translocation of Daxx (death associated protein) in ventral midbrain and striatum, attenuating dopaminergic cell loss. R-LA induced significant reductions in markers of oxidative modifications in transgenic AD mice model, significantly decreasing $\mathrm{HO}-1$ and protein-bound $\mathrm{HNE}$ levels (Siedlak et al., 2009). Inman et al. (2013) analyzed the effect of LA in the DBA/2J mouse model of glaucoma. The results showed that after 4 and 11 months of dietary LA, respectively, LA treatment increased antioxidant genes and protein expression, protected retinal ganglion cell (RGC), and improved retrograde transport. Dietary therapy also reduced lipid peroxidation, protein nitrosylation, and DNA oxidation in a retina model of glaucoma.

Accumulation of metal ions also has been associated with increased oxidative stress related with aging and neurodegenerative disorders. Suh et al. (2005) showed that LA supplementation can modulate age-related cortical iron accumulation, acting as metal chelator, thereby ameliorating age-associated oxidative stress. However, Liu et al. (2002b) showed that high concentrations of iron and copper found in old rats were not significantly decreased with LA supplementation.

There are multiple cell death mechanisms implicated in neurodegeneration. Apoptosis is a highly controlled cellular process that can be activated by two pathways: extrinsic, which is a receptor-mediated pathway, and intrinsic, which is mediated by signals from the mitochondria. Both pathways culminate at cleavage-dependent activation of aspartate-specific effector caspases (caspases-3, 6, and 7). Cui et al. (2006), using chronic systemic exposure of d-galactose in an aging model observed that a treatment with LA decreased caspase-3 protein levels and neuronal apoptosis, ameliorating neurodegeneration in the hippocampus. Manda et al. (2007), demonstrated that LA pretreatment protected against radiation. They observed that 
TABLE 1 | Summary of studies testing the effects of LA on behavioral parameters in animal models.

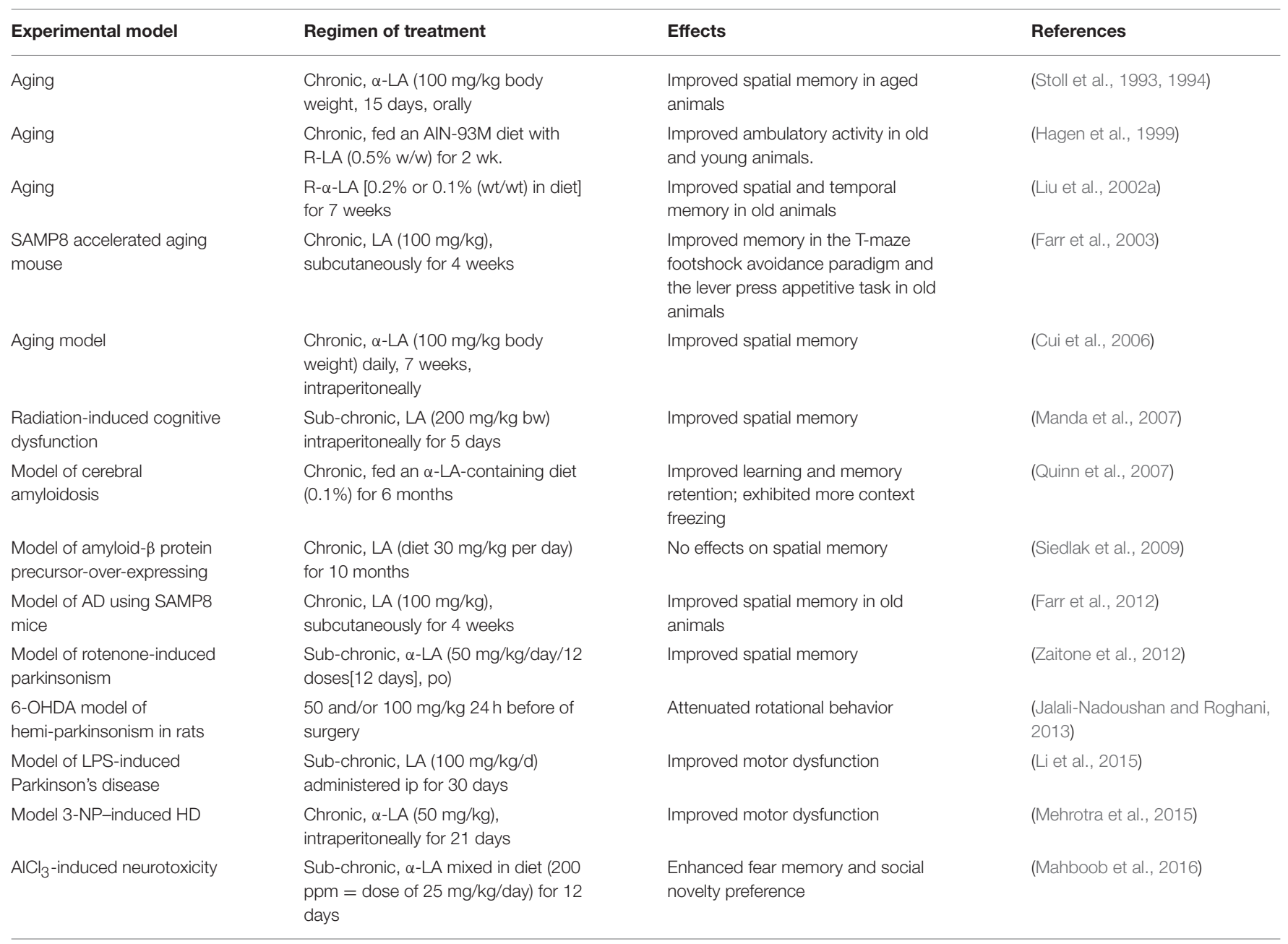

3-NP, 3-nitropropionic acid; AD, Alzheimer's disease; AlCl3, Aluminum chloride; HD, Huntington's disease; LPS, lipolysaccharide; 6-OHDA, 6-hydroxydopamine.

pre-treatment with LA prevented radiation-induced decreases of total, nonprotein and protein-bound sulfhydryl (T-SH, NP-SH, and $\mathrm{PB}-\mathrm{SH}$ ) levels in the cerebellum. Moreover, LA treatment also improved the cytoarchitecture of cerebellum, increasing the number of intact Purkinje cells and granular cells when compared to untreated irradiated mice.

Mehrotra et al. (2015) evaluated the effects of LA on mitochondrial dysfunctions in the 3-NP induced model of HD. The results showed that LA decreased malondialdehyde, protein carbonyls, reactive oxygen species and nitrite levels, and increased Mn-superoxide dismutase and CAT activity. They also found that LA improved histological and biochemical alterations, such as decreased cytosolic cytochrome c levels, caspase-3 and -9 activity and expression of apoptotic proteins (AIF, Bim, Bad, and Bax), suggesting its therapeutic efficacy in HD. LA improved activity of enzymes from the mitochondrial respiratory chain, altered cytochrome levels, increased histochemical staining of complex-II and IV, increased in-gel activity of complex$\mathrm{I}$ to $\mathrm{V}$, and increased mRNA expression of respiratory chain complexes.
Stoll et al. (1993) investigated the effect of LA on NMDA Receptor deficits in old female NMRI mice. The results showed that LA improved age-related NMDA receptor deficits $\left(B_{\max }\right)$. No changes were observed regarding muscarinic, benzodiazepine, and $\alpha_{2}$-adrenergic receptor deficiencies. Thus, the authors concluded that LA-induced memory improving effects may be related to partial reparation of NMDA receptor deficits that accompany aging.

A loss of dopaminergic neurons is particularly relevant to $\mathrm{PD}$, in which genetic and environmental factors are involved (Di Domenico et al., 2015; Li et al., 2015). Jalali-Nadoushana and Roghania using a rat model of hemi-parkinsonism (6-OHDA) found that LA prevented neuronal loss on the left side of the substantia nigra pars compacta (SNpc) (Jalali-Nadoushan and Roghani, 2013). In a study using the LPS-induced inflammatory PD model, Li et al. (2015) demonstrated that LA administration protected against dopaminergic neuron loss. Zaitone et al. (2012) observed that LA induced an increase in the number of neurons in the SNpc in rotenone parkinsonian rats. Li et al. (2015) reported that in addition to protecting against dopaminergic 
TABLE 2 | Summary of in vivo studies testing cellular and molecular effects of $L A$.

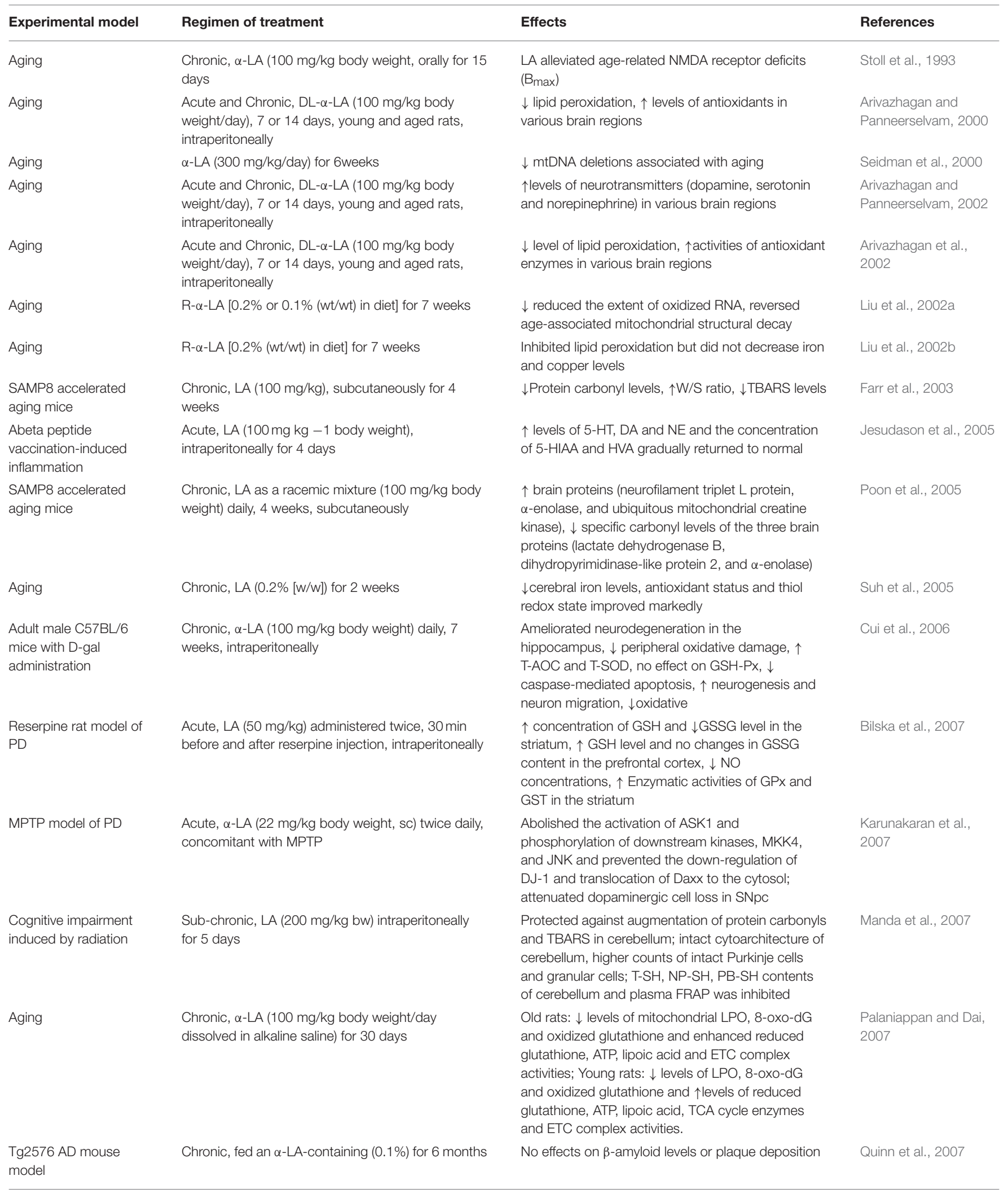


TABLE 2 | Continued

\begin{tabular}{ll}
\hline Experimental model & Regimen of treatment \\
\hline Aging & Acute, LA (10, 20 or $30 \mathrm{mg} / \mathrm{kg}$, i.p.), $24 \mathrm{~h}$ \\
& \\
$\begin{array}{l}\text { Tg2576 AD mouse } \\
\text { model }\end{array}$ & Chronic, LA (diet $30 \mathrm{mg} / \mathrm{kg}$ per day) for 10 months
\end{tabular}
model

Effects

$\downarrow$ lipid peroxidation level, no alteration was observed in SOD activity, $\uparrow$ DA and NE, $\downarrow 5-H T$ and their metabolites 5-HIAA, the metabolites (DOPAC and HVA) did not differ in hippocampus $\downarrow$ expression of $\mathrm{HO}-1$ and protein-bound HNE, $\downarrow$ HO- 1 around amyloid plaques, $\downarrow$ protein-bound HNE expression surrounding A $\beta$ plaques, Redox active iron accumulation was specifically co-localized with $A \beta$ plaques in the hippocampus and cortical regions

Pilocarpine-induced seizures

$\mathrm{AlCl}_{3}$ rat model of $\mathrm{AD}$

SAMP8 accelerated aging mice Rotenone rat model of PD

Acute, LA (20 mg/kg, ip) for $30 \mathrm{~min}$

Chronic, $\alpha$-LA (100 mg/kg/day for 3 months) after $\mathrm{AlCl}_{3}$ (100 mg/kg b.wt/day for 4 months), orally. Chronic, LA (100 mg/kg), subcutaneously for 4 weeks

Sub-chronic, $\alpha$-LA (50 mg/kg/day/12 doses[12 days], po)

DBA/2J mouse model of glaucoma

Unilateral intrastriatal 6-OHDA-lesioned rats $3 \times \mathrm{Tg}-\mathrm{AD}$

Arsenic-dichlorvos exposed rats Lipolysaccharide (LPS)-induced inflammatory PD model

3-NP-induced HD model of $\downarrow$ lipid peroxidation and nitrite concentrations and $\uparrow S O D, C A T$ and GPx activities in striatum

$\downarrow$ AChE activity, $\downarrow$ inflammation, $\uparrow$ neuronal and regeneration features

$\uparrow \mathrm{GSH}, \downarrow \mathrm{MDA}, \downarrow \mathrm{GPx}$

$\uparrow$ striatal dopamine level, no effect on striatal ATP

level, $\downarrow$ level of lipid peroxides and $\downarrow$ protein carbonyls in rat brains, $\uparrow$ tissue GSH, improved injury to mtDNA and normalization of the mtDNA content, $\uparrow$ percentage of SNpc dopaminergic neurons, $\uparrow$ number of Nissl stained neurons $\uparrow a n t i o x i d a n t$ gene and protein expression, 个protection of RGCs and improved retrograde transport, $\downarrow$ lipid peroxidation, $\downarrow$ protein nitrosylation, $\downarrow$ DNA oxidation in the prevention and intervention paradigms.

Prevented loss of SNC neurons, $\downarrow$ levels of MDA and nitrite

$\uparrow$ brain glucose uptake; $\uparrow$ in the total GLUT3 and GLUT4 in the old mice; activation of the insulin receptor substrate and of the PI3K/Akt signaling pathway; changes in synaptic function $(\uparrow / / O)$ and LTP.

Oxidative stress and cholinergic dysfunction was protected

Protected dopaminergic neurons loss, $\downarrow \alpha$-synuclein accumulation in the substantia nigra, inhibited the activation of NF-KB and expression of pro-inflammatory molecules in M1 microglia

Restored respiratory chain enzyme activities, CAT activity was improved, normalized of mitochondrial appearance, stimulated the repair of mitochondrial membranes and restored functionality to impaired mitochondria, $\downarrow$ lipid peroxidation, $\downarrow$ protein carbonyls, $\downarrow$ ROS and nitrite levels, $\downarrow$ cytosolic levels of cytochrome c, $\downarrow$ activities of caspase-3 and 9. Improves the expression of muscarinic receptors (M1 and M2) and choline acetyltransferase
$60 \mathrm{mg} / \mathrm{kg}$ body weight (bw)/day for the intervention study and $100 \mathrm{mg} / \mathrm{kg}$ bw/day for the prevention study in diet for 24 months

50 and $/$ or $100 \mathrm{mg} / \mathrm{kg} 24 \mathrm{~h}$ before surgery

Chronic, LA (0.23\% W/v in drinking water) for 4 weeks

Chronic, $\alpha$-LA (50 mg/kg/day for 10 months), orally.

Sub-chronic, LA (100 mg/kg/d) administered ip for 30 days

Chronic, $\alpha$-LA (50 mg/kg), intraperitoneally for 21 days

Sub-chronic, $\alpha$-LA mixed in diet $(200$ ppm $=$ dose of $25 \mathrm{mg} / \mathrm{kg} /$ day) for 12 days
References

Ferreira et al., 2009

Siedlak et al., 2009

Militao et al., 2010

Ahmed, 2012

Farr et al., 2012

Zaitone et al., 2012

Inman et al., 2013

Jalali-Nadoushan and Roghani, 2013

Sancheti et al., 2013

Dwivedi et al., 2014

Li et al., 2015

Mehrotra et al., 2015

Mahboob et al., 2016
$\mathrm{AlCl}_{3}$-induced
neurotoxicity mouse model

3-NP, 3-nitropropionic acid; 5-HIAA, 5-hydroxyindoleacetic acid; 5-HT, 5-hydroxytryptamine; 5-HT, serotonina; 6-OHDA, 6-hydroxydopamine; 8-oxo-dG, monoclonal anti-8hydroxyguanine; $A C h E$, acetylcholinesterase; $A D$, Alzheimer's disease; AICl, Aluminum chloride: ASK1, apoptosis signal regulating kinase 1; AB, amyloid- $\beta$ fibrils; CAT, catalase activity; DA, dopamine; D-gal, D-galactose; DOPAC, 3,4-hydroxyphenylacetic acid; ETC, electron transport chain; FRAP, ferric reducing power; GLUT3, glucose transporter 3; GLUT4, glucose transporter 4; GPx, glutathione peroxidase; GSH, reduced glutathione; GSH-Px, glutathione peroxidase; GSSG, glutathione disulfide; GST, glutathione-S-transferase; HNE, 4-hydroxynonenal; HO-1, heme oxygenase-1; HVA, homovanillic acid; I/O, input/output; JNK, Jun N-terminal kinase; LPO, lipid peroxidation; LPS, Lipolysaccharide; LTP, Iong term potentiation; M1, Type 1 macrophages/microglia; M2, Type 2 macrophages/microglia; MDA, malondialdehyde; MKK4, mitogen-activated protein kinase kinase 4; MPTP, 1-methyl-4phenyl-1, 2, 3, 6-tetrahydropyridine; mtDNA, mitochondrial DNA; NE, norepinephrine; NF-kB, nuclear factor-kB; NMDA receptor, N-methyl-D-aspartate receptor; NO, nitric oxide; PD, Parkinson's disease; RGC, retinal ganglion cell; ROS, reactive oxygen species; SNC, substantia nigra pars compacta; SNpc, substantia nigra pars compacta; SOD, total superoxide dismutase; T-AOC, total antioxidative capabilities; TBARS, thiobarbituric acid reactive substance; T-SOD, total superoxide dismutase; W/S, weakly immobilized/strongly immobilized. 
neuron loss, LA also decreased $\alpha$-synuclein deposits in the substantia nigra (SN). Moreover, the authors showed that LA inhibited the stimulation of nuclear factor- $\kappa \mathrm{B}(\mathrm{NF}-\kappa \mathrm{B})$ and expression of pro-inflammatory molecules in M1 microglia. Zaitone et al. (2012) also investigated the effect of LA on mitochondrial DNA (mtDNA) integrity and quantity in the rotenone model of $\mathrm{PD}$. The results showed that LA significantly decreased rotenone-induced mtDNA damage.

Liu et al. (2002a), examined the effects of LA on mitochondrial structure, and neurodegeneration in the hippocampus, and oxidative damage to nucleic acids in the hippocampus and cortex of aged rats. Dietary administration of LA significantly reduced oxidized RNA levels and reversed mitochondrial structural deterioration induced by aging in the hippocampus. Dwivedi et al. (2014) investigated the protective efficacy of LA against coexposure to arsenic-dichlorvos in rats. The results indicated that arsenic and dichlorvos induced oxidative stress and cholinergic dysfunction in brain, which was significantly protected by the supplementation with LA.

Seidman et al. (2000) indicated that age-associated mitochondrial impairment may be hampered by LA administration. Their results showed that mtDNA deletions associated with aging were reduced by LA and this effect appeared to be related to the mitochondrial capacity to protect and repair mtDNA against age-induced injury. Palaniappan and Dai (2007) investigated the effect of LA administration to aged rats and verified a reduction of mitochondrial lipid peroxidation, 8-oxo-dG and oxidized glutathione (GSSG) and increased GSH, ATP, and electron transport chain (ETC) complex activities in the brain.

The SAMP8 mouse strain is an experimental model that displays increased oxidative stress accompanied by memory decline associated to a rapid aging process. Proteomic analyses were used to examine differential protein expression and/or protein oxidative changes in brain samples from aged SAMP8 mice. In order to determine the mechanisms underlying LAinduced reversion of memory deficits exhibited by SAMP8 mice, Poon et al. (2005) analyzed the expression and specific carbonylation of proteins in brains from 12-month-old SAMP8 mice that received LA or vehicle. The levels of three proteins (neurofilament triplet L protein, a-enolase, and ubiquitous mitochondrial creatine kinase) were significantly increased, while protein carbonylation was reduced in lactate dehydrogenase $\mathrm{B}$, dihydropyrimidinase-like protein 2 , and a-enolase in aged SAMP8 mice that received LA, suggesting that, in addition to improving learning and memory, LA also can restore specific proteins in aged SAMP8 mouse brain.

Evidence indicates that deregulation in neurotransmitter systems, including decreased levels of neurotransmitters, decline in the number of receptors, and lower responsiveness to neurotransmitters can be key features of neurological disorders (Payton et al., 2005; Fidalgo et al., 2013). Arivazhagan and Panneerselvam (2002) investigated the effect of LA on levels of neurotransmitters (dopamine, serotonin, and norepinephrine), and showed that LA treatment can improve neurotransmitter function in models of neurodegenerative diseases. Jesudason et al. (2005) investigated the effect LA on levels of neurotransmitters in a model of $\mathrm{AD}$ by $\mathrm{A} \beta$ amyloid vaccination. The results showed that AD mice treated with LA exhibited enhanced levels of serotonin, dopamine, and norepinephrine, and the concentration of metabolites 5-hydroxyindole acetic acid (5HIAA) and homovanillic acid (HVA) gradually returned to normal.

Ahmed (2012) explored the effect of LA on brain acetylcholinesterase (AChE) activity. The authors demonstrated that LA can ameliorate neurological injury related to $A \beta$ and $\mathrm{Al}$ excess, by significantly restoring $\mathrm{AChE}$ activity. In addition, the authors showed that the treatment with LA restored the parameters of total homocysteine (tHcy), insulin, insulin like growth factor-1 (IGF-1), interlukin-1 $\beta$ (IL-1 $\beta$ ) and tumor necrosis factor- $\alpha$ (TNF- $\alpha$ ). Mahboob et al. (2016), analyzed the effects of $\mathrm{LA}$ in $\mathrm{AlCl}_{3}$ - model of neurodegeneration, demonstrating its capacity in ameliorating cognitive functions and enhancing cholinergic system functions. LA treatment increased the expression of muscarinic receptor genes M1, M2 and choline acetyltransferase (ChaT) relative to $\mathrm{AlCl}_{3}$-treated group.

There are many studies examining the neuroprotective actions of LA using in vitro models of neurodegeneration (Tirosh et al., 1999; Li et al., 2013; Xing et al., 2015), most of which focus on AD. For example, Ono et al. (2006) investigated the effects of LA and its reduced form, DHLA, on the formation, extension, and destabilization of $\beta$-amyloid fibrils (fA $\beta$ ). The results showed that both LA and DHLA inhibited fA $\beta$ formation from amyloid $\beta$, as well as their expansion, and undermined preformed fA $\beta$ s in a dose dependent manner. Lovell et al. (2003) also studied the effects of LA and DHLA in neuronal cultures challenged with amyloid $\beta$-peptide (A $\beta$ 25-35), and observed that DHLA, but not LA, significantly protected against neurotoxicity induced by amyloid $\beta$-peptide and iron/hydrogen peroxide $\left(\mathrm{Fe} / \mathrm{H}_{2} \mathrm{O}_{2}\right)$.

In $\beta$-amyloid-intoxicated C6 glioma cells, LA increased cell viability and MnSOD expression. The increased GSSH and decreased GSH mitochondrial levels induced by $\mathrm{A} \beta$ were reversed by treatment with LA (Xing et al., 2015). In addition, LA protected cortical neurons against $A \beta$ peptide- and hydrogen peroxide-induced damage, suggesting that the neuroprotective effects were partly related to $\mathrm{PKB} / \mathrm{Akt}$ signaling pathway stimulation (Zhang et al., 2001).

The study by Deuther-Conrad et al. (2001) showed that the advanced glycation end products (AGE)-induced increases in oxidized glutathione were inhibited by R-LA in SH-SY5Y human neuroblastoma cells, indicating that AGE-mediated depletion of reduced glutathione follows the production of superoxide and hydrogen peroxide. de Arriba et al. (2003) investigated the effect of R-LA, in the same types of cells, on AGE accumulation, and found that AGE-induced metabolic changes were diminished by R-LA. Tirosh et al. (1999) showed that LA protected HT4 neuronal cells against glutamate-induced cytotoxicity, by inhibiting intracellular GSH depletion, and canceled the buildup of intracellular peroxide levels following the glutamate exposure.

Kamarudin et al. (2014) showed that R-LA ameliorated glutathione over glutathione disulfide ratio, decreased intracellular ROS levels and increased mitochondrial membrane potential in NG108-15 cells. In addition, R-LA stimulated the production of an anti-inflammatory cytokine, IL-10, inactivating

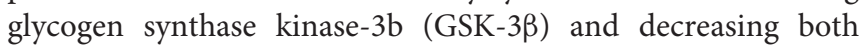


$\mathrm{Bax} / \mathrm{Bcl} 2$ and $\mathrm{Bax} / \mathrm{Bcl}-\mathrm{xL}$ ratios. Suppression of NF-к $\beta$ p65 translocation and production of proinflammatory cytokines (IL-6 and TNF- $\alpha$ ) followed inhibition of cleaved caspase-3. Yamada et al. (2011) investigated the effects of different isomers of LAs (racemate, R-LA, and S-LA) in human neuroblastoma SH-SY5Y cells. They showed that all types of LAs were effective in preventing cell death. R-LA and S-LA also enhanced expression of genes related to anti-oxidative response such as heme oxygenase-1 (HO-1) and phase II detoxification enzymes such as NAD(P)H:Quinone Oxidoreductase 1 (NQO1).

Other studies evaluated the effect of LA on in vitro model of PD. Li et al. (2013) showed that pretreatment with LA significantly prevented against apoptosis of PC12 cells elicited by MPP+, and inhibited intercellular ROS levels and mitochondrial transmembrane permeability, thereby protecting dopaminergic neuronal cells against oxidative damage. Moreover, Zhang et al. (2010) demonstrated that R-LA hindered rotenoneinduced mitochondrial dysfunction, oxidative damage, and $\alpha$-synuclein and ubiquitin deposition in SK-N-MC human neuroblastoma cells.

\section{Involvement of LA in the Regulation of Cellular Signaling Pathways}

LA has been proposed to exert a modulatory control on the cellular redox status. Due to its ability to be interconverted in one of its two forms-i.e., thiol, the reduced form and disulfide, the oxidized form-LA can regulate cellular redox environment by interacting with redox couples such as glutathione/glutathione disulfide, cysteine/cystine, and thioredoxin (Packer and Cadenas, 2011). LA has been described to regenerate other antioxidants, such as vitamin $\mathrm{C}$ and $\mathrm{E}$, to increase GSH levels, and to provide modulation of proteins and transcription factors (Packer et al., 1995). Extracellular redox state is also regulated by LA, once its reduced form, DHLA, can interact with cystine, reducing it to cysteine, thereby stimulating its uptake by the cell, which in turn stimulates GSH synthesis (Han et al., 1997). Owing to the role played by LA in the regulation of thiol/disulfide redox couples, LA can be viewed as regulator of cell signaling and gene expression.

PI3K/Akt signaling pathway, critical to the regulation of cell growth, proliferation, differentiation, survival, and metabolism, has been shown to be modulated by LA. For instance, Jiang et al. (2013) have demonstrated that age-associated imbalance of PI3K/Akt was restored by LA treatment for 3 weeks in the drinking water in rats. LA treatment significantly increased Akt phosphorylation and lead to a recovery in the ratio pJNK/pAkt in cortex of aged rats. Prevention of sevoflurane-induced apoptosis by LA was accomplished through recovery of Akt and GSK3$\beta$ phosphorylation levels in the hippocampus (Ma et al., 2016). By activating PI3K/Akt signaling pathway, LA, administered for 3 days, was able to ameliorate cerebral ischemia and reperfusion-induced damage in adult rats (Dong et al., 2015). Sancheti et al. (2013) showed that LA increases brain glucose uptake and activates the insulin receptor substrate and the $\mathrm{PI} 3 \mathrm{~K} / \mathrm{Akt}$ signaling pathway in a triple transgenic mouse model of $\mathrm{AD}(3 \mathrm{xTg}-\mathrm{AD})$, reversing the impaired synaptic plasticity and increasing input/output (I/O) and long-term potentiation (LTP).

\section{CONCLUDING REMARKS}

Compelling evidence indicates that LA displays memoryameliorating properties in a variety of experimental models of neurodegenerative diseases, as well as in memory decline associated with aging in rodents. Studies aiming to assess the neuroprotective effects of LA on behavioral outcomes showed that LA can reduce memory deficits in different behavioral paradigms on AD (Quinn et al., 2007; Farr et al., 2012), HD (Mehrotra et al., 2015), oxidative stress (Stoll et al., 1993, 1994; Liu et al., 2002a; Farr et al., 2003; Manda et al., 2007), and age-associated cognitive dysfunction (Cui et al., 2006; Mahboob et al., 2016) models. In humans, two studies in AD patients have supported the positive cognitive effects of LA (Hager et al., 2001, 2007).

Many studies reported beneficial effects of LA in the rat brain or neuronal cell cultures, using different molecular markers of oxidative stress, such as reduction in the levels of lipid peroxides and protein carbonyls, recycling endogenous antioxidants such as vitamin $\mathrm{C}$ and $\mathrm{E}$, increasing glutathione levels (Packer et al., 1997; Di Domenico et al., 2015; Mehrotra et al., 2015), inhibiting free radical formation, chelating transition metal ions such as iron, thus reducing its bioaccumulation in the brain (Moini et al., 2002; Shay et al., 2009; Rochette et al., 2013). LA was also shown to display anti-inflammatory properties (Deuther-Conrad et al., 2001; Li et al., 2015), and affect cell death. In vivo and in vitro studies showed that LA ameliorated neurodegeneration in the hippocampus, decreasing neuronal apoptosis and caspase-3 protein levels, supporting a neuroprotective role mediated by the mitochondrial cell death pathway. These effects suggest that LA is able to improve mitochondrial dysfunctions. Interestingly, in addition to decreasing neuronal cell death, LA also inhibited fA $\beta$ formation from amyloid $\beta$-protein, ameliorating the neurological damage induced by $\mathrm{A} \beta$, and significantly restored $\mathrm{AChE}$ activity. This evidence suggests that LA presents a potential role in enhancing cholinergic and cognitive functions. These neuroprotective effects may be related to the properties of LA in ameliorating memory loss associated to neurodegenerative diseases.

Remarkably, LA was able to reverse age-associated glutamatergic NMDA receptor deficits (Stoll et al., 1993), which might be centrally related to LA memory-improving effects. LA was also shown to improve the function of neurotransmitter systems, including dopamine, serotonin, and norepinephrine. Taken together, these findings provide evidence that LA can reverse loss of neurotransmitters, their receptors and responsiveness to neurotransmitters, which can underlie its effects on cognitive functions.

In summary, this review has described and discussed relevant studies investigating the effects of LA on cognition as well as its cellular and molecular effects, aiming to improve the understanding of the therapeutic potential of LA in memory loss during aging and patients suffering from neurodegenerative disorders. Although the mechanisms of action of LA are not fully understood, multiple pathways are likely to be involved in its neuroprotective properties. The memory-improving effects and neuroprotective actions of LA support its use as an adjuvant treatment for neurodegenerative disorders. 


\section{AUTHOR CONTRIBUTIONS}

PM has performed literature search and has written the first draft of the manuscript. NS has extensively revised and contributed in writing the final version of the manuscript.

\section{REFERENCES}

Abadi, A., Crane, J. D., Ogborn, D., Hettinga, B., Akhtar, M., Stokl, A., et al. (2013). Supplementation with alpha-lipoic acid, CoQ10, and vitamin E augments running performance and mitochondrial function in female mice. PLOS ONE 8:e60722. doi: 10.1371/journal.pone.0060722

Ahmed, H. H. (2012). Modulatory effects of vitamin E, acetyl-L-carnitine and alpha-lipoic acid on new potential biomarkers for Alzheimer's disease in rat model. Exp. Toxicol. Pathol. 64, 549-556. doi: 10.1016/j.etp.2010.11.012

Aliev, G., Liu, J., Shenk, J. C., Fischbach, K., Pacheco, G. J., Chen, S. G., et al. (2009). Neuronal mitochondrial amelioration by feeding acetyl-Lcarnitine and lipoic acid to aged rats. J. Cell Mol. Med. 13, 320-333. doi: 10.1111/j.1582-4934.2008.00324.X

Arivazhagan, P., and Panneerselvam, C. (2000). Effect of DL-alpha-lipoic acid on neural antioxidants in aged rats. Pharmacol. Res. 42, 219-222. doi: $10.1006 /$ phrs.2000.0679

Arivazhagan, P., and Panneerselvam, C. (2002). Neurochemical changes related to ageing in the rat brain and the effect of DL-alpha-lipoic acid. Exp. Gerontol. 37, 1489-1494. doi: 10.1016/S0531-5565(02)00122-5

Arivazhagan, P., Ramanathan, K., and Panneerselvam, C. (2001a). Effect of DL-alpha-lipoic acid on the status of lipid peroxidation and antioxidants in mitochondria of aged rats. J. Nutr. Biochem. 12, 2-6. doi: 10.1016/S0955-2863(00)00138-8

Arivazhagan, P., Ramanathan, K., and Panneerselvam, C. (2001b). Effect of DLalpha-lipoic acid on glutathione metabolic enzymes in aged rats. Exp. Gerontol. 37, 81-87. doi: 10.1016/S0531-5565(01)00147-4

Arivazhagan, P., Shila, S., Kumaran, S., and Panneerselvam, C. (2002). Effect of DL-alpha-lipoic acid on the status of lipid peroxidation and antioxidant enzymes in various brain regions of aged rats. Exp. Gerontol. 37, 803-811. doi: 10.1016/S0531-5565(02)00015-3

Bagh, M. B., Thakurta, I. G., Biswas, M., Behera, P., and Chakrabarti, S. (2011). Age-related oxidative decline of mitochondrial functions in rat brain is prevented by long term oral antioxidant supplementation. Biogerontology 12, 119-131. doi: 10.1007/s10522-010-9301-8

Bast, A., and Haenen, G. R. (2003). Lipoic acid: a multifunctional antioxidant. Biofactors 17, 207-213. doi: 10.1002/biof.5520170120

Beal, M. F. (2003). Bioenergetic approaches for neuroprotection in Parkinson's disease. Ann. Neurol. 53 (Suppl. 3), S39-S47; Discussion S-8. doi: 10.1002/ana.10479

Bilska, A., Dubiel, M., Sokolowska-Jezewicz, M., Lorenc-Koci, E., and Wlodek, L. (2007). Alpha-lipoic acid differently affects the reserpine-induced oxidative stress in the striatum and prefrontal cortex of rat brain. Neuroscience 146, 1758-1771. doi: 10.1016/j.neuroscience.2007.04.002

Bishop, N. A., Lu, T., and Yankner, B. A. (2010). Neural mechanisms of ageing and cognitive decline. Nature 464, 529-535. doi: 10.1038/nature08983

Bustamante, J., Lodge, J. K., Marcocci, L., Tritschler, H. J., Packer, L., and Rihn, B. H. (1998). Alpha-lipoic acid in liver metabolism and disease. Free Radic. Biol. Med. 24, 1023-1039. doi: 10.1016/S0891-5849(97)00371-7

Chen, X., Guo, C., and Kong, J. (2012). Oxidative stress in neurodegenerative diseases. Neural Regen. Res. 7, 376-385. doi: 10.3969/j.issn.1673-5374.2012.05.009

Cremer, D. R., Rabeler, R., Roberts, A., and Lynch, B. (2006a). Long-term safety of alpha-lipoic acid (ALA) consumption: a 2-year study. Regul. Toxicol. Pharmacol. 46, 193-201. doi: 10.1016/j.yrtph.2006.06.003

Cremer, D. R., Rabeler, R., Roberts, A., and Lynch, B. (2006b). Safety evaluation of alpha-lipoic acid (ALA). Regul. Toxicol. Pharmacol. 46, 29-41. doi: 10.1016/j.yrtph.2006.06.004

Cui, X., Zuo, P., Zhang, Q., Li, X., Hu, Y., Long, J., et al. (2006). Chronic systemic $\mathrm{D}$-galactose exposure induces memory loss, neurodegeneration, and oxidative

\section{FUNDING}

This research was supported by the National Council for Scientific and Technological Development (CNPq; grant number 308290/2015-1 to NS).

damage in mice: protective effects of R-alpha-lipoic acid. J. Neurosci. Res. 84, 647-654. doi: 10.1002/jnr.20899

De Araujo, D. P., Lobato Rde, F., Cavalcanti, J. R., Sampaio, L. R., Araujo, P. V., Silva, M. C., et al. (2011). The contributions of antioxidant activity of lipoic acid in reducing neurogenerative progression of Parkinson's disease: a review. Int. J. Neurosci. 121, 51-57. doi: 10.3109/00207454.2010.535934

de Arriba, S. G., Loske, C., Meiners, I., Fleischer, G., Lobisch, M., Wessel, K., et al (2003). Advanced glycation endproducts induce changes in glucose consumption, lactate production, and ATP levels in SH-SY5Y neuroblastoma cells by a redox-sensitive mechanism. J. Cereb. Blood Flow Metab. 23, 1307-1313. doi: 10.1097/01.WCB.0000090622.86921.0E

Deuther-Conrad, W., Loske, C., Schinzel, R., Dringen, R., Riederer, P., and Munch, G. (2001). Advanced glycation endproducts change glutathione redox status in SH-SY5Y human neuroblastoma cells by a hydrogen peroxide dependent mechanism. Neurosci. Lett. 312, 29-32. doi: 10.1016/S0304-3940(01)02174-7

Di Domenico, F., Barone, E., Perluigi, M., and Butterfield, D. A. (2015). Strategy to reduce free radical species in Alzheimer's disease: an update of selected antioxidants. Expert Rev. Neurother. 15, 19-40. doi: $10.1586 / 14737175.2015 .955853$

Dong, Y., Wang, H., and Chen, Z. (2015). Alpha-Lipoic Acid Attenuates Cerebral Ischemia and Reperfusion Injury via Insulin Receptor and PI3K/AktDependent Inhibition of NADPH Oxidase. Int. J. Endocrinol. 2015:903186. doi: $10.1155 / 2015 / 903186$

Dwivedi, N., Flora, G., Kushwaha, P., and Flora, S. J. (2014). Alphalipoic acid protects oxidative stress, changes in cholinergic system and tissue histopathology during co-exposure to arsenic-dichlorvos in rats. Environ. Toxicol. Pharmacol. 37, 7-23. doi: 10.1016/j.etap.2013. 10.010

Farr, S. A., Poon, H. F., Dogrukol-Ak, D., Drake, J., Banks, W. A., Eyerman, E., et al. (2003). The antioxidants alpha-lipoic acid and Nacetylcysteine reverse memory impairment and brain oxidative stress in aged SAMP8 mice. J. Neurochem. 84, 1173-1183. doi: 10.1046/j.1471-4159.2003.0 1580.x

Farr, S. A., Price, T. O., Banks, W. A., Ercal, N., and Morley, J. E. (2012). Effect of alpha-lipoic acid on memory, oxidation, and lifespan in SAMP8 mice. J. Alzheimers Dis. 32, 447-455. doi: 10.3233/JAD-2012-120130

Ferreira, B., Mendes, F., Osorio, N., Caseiro, A., Gabriel, A., and Valado, A. (2013). Glutathione in multiple sclerosis. Br. J. Biomed. Sci. 70, 75-79. doi: 10.1080/09674845.2013.11669939

Ferreira, P. M., Militao, G. C., and Freitas, R. M. (2009). Lipoic acid effects on lipid peroxidation level, superoxide dismutase activity and monoamines concentration in rat hippocampus. Neurosci. Lett. 464, 131-134. doi: 10.1016/j.neulet.2009.08.051

Fidalgo, S., Ivanov, D. K., and Wood, S. H. (2013). Serotonin: from top to bottom. Biogerontology 14, 21-45. doi: 10.1007/s10522-012-9406-3

Ghibu, S., Richard, C., Vergely, C., Zeller, M., Cottin, Y., and Rochette, L. (2009). Antioxidant properties of an endogenous thiol: Alpha-lipoic acid, useful in the prevention of cardiovascular diseases. J. Cardiovasc. Pharmacol. 54, 391-398. doi: 10.1097/FJC.0b013e3181be7554

Go, Y. M., and Jones, D. P. (2017). Redox theory of aging: implications for health and disease. Clin. Sci. (Lond.) 131, 1669-1688. doi: 10.1042/CS20160897

Gonzalez, P., da Costa, V. C., Hyde, K., Wu, Q., Annunziata, O., Rizo, J., et al. (2014). Bimodal-hybrid heterocyclic amine targeting oxidative pathways and copper mis-regulation in Alzheimer's disease. Metallomics 6, 2072-2082. doi: 10.1039/C4MT00161C

Hagen, T. M., Ingersoll, R. T., Lykkesfeldt, J., Liu, J., Wehr, C. M., Vinarsky, V., et al. (1999). (R)-alpha-lipoic acid-supplemented old rats have improved mitochondrial function, decreased oxidative damage, and increased metabolic rate. FASEB J. 13, 411-418. 
Hager, K., Kenklies, M., McAfoose, J., Engel, J., and Munch, G. (2007). Alpha-lipoic acid as a new treatment option for Alzheimer's disease-a 48 months follow-up analysis. J. Neural Transm. Suppl. 72, 189-193.

Hager, K., Marahrens, A., Kenklies, M., Riederer, P., and Munch, G. (2001). Alphalipoic acid as a new treatment option for Alzheimer type dementia. Arch. Gerontol. Geriatr. 32, 275-282. doi: 10.1016/S0167-4943(01)00104-2

Han, D., Handelman, G., Marcocci, L., Sen, C. K., Roy, S., Kobuchi, H., et al. (1997). Lipoic acid increases de novo synthesis of cellular glutathione by improving cystine utilization. Biofactors 6, 321-338. doi: 10.1002/biof.5520060303

Hiller, S., DeKroon, R., Hamlett, E. D., Xu, L., Osorio, C., and Robinette, J., et al. (2016). Alpha-lipoic acid supplementation protects enzymes from damage by nitrosative and oxidative stress. Biochim. Biophys. Acta 1860(1 Pt A), 36-45. doi: 10.1016/j.bbagen.2015.09.001

Holmquist, L., Stuchbury, G., Berbaum, K., Muscat, S., Young, S., Hager, K., et al. (2007). Lipoic acid as a novel treatment for Alzheimer's disease and related dementias. Pharmacol. Ther. 113, 154-164. doi: 10.1016/j.pharmthera.2006.07.001

Inman, D. M., Lambert, W. S., Calkins, D. J., and Horner, P. J. (2013). alpha-Lipoic acid antioxidant treatment limits glaucoma-related retinal ganglion cell death and dysfunction. PLoS ONE 8:e65389. doi: 10.1371/journal.pone.0065389

Irwin, M. H., Moos, W. H., Faller, D. V., Steliou, K., and Pinkert, C. A. (2016). Epigenetic treatment of neurodegenerative disorders: Alzheimer and Parkinson Diseases. Drug Dev. Res. 77, 109-123. doi: 10.1002/ddr.21294

Jalali-Nadoushan, M., and Roghani, M. (2013). Alpha-lipoic acid protects against 6-hydroxydopamine-induced neurotoxicity in a rat model of hemiparkinsonism. Brain Res. 1505, 68-74. doi: 10.1016/j.brainres.2013.01.054

Jesudason, E. P., Masilamoni, J. G., Kirubagaran, R., Davis, G. D., and Jayakumar, R. (2005). The protective role of DL-alpha-lipoic acid in biogenic amines catabolism triggered by Abeta amyloid vaccination in mice. Brain Res. Bull. 65, 361-367. doi: 10.1016/j.brainresbull.2005.01.010

Jiang, T., Yin, F., Yao, J., Brinton, R. D., and Cadenas, E. (2013). Lipoic acid restores age-associated impairment of brain energy metabolism through the modulation of Akt/JNK signaling and PGC1alpha transcriptional pathway. Aging Cell 12, 1021-1031. doi: 10.1111/acel.12127

Jones, D. P., and Sies, H. (2015). The Redox Code. Antioxid Redox Signal 23, 734-746. doi: 10.1089/ars.2015.6247

Kamarudin, M. N., Mohd Raflee, N. A., Hussein, S. S., Lo, J. Y., Supriady, H., and Abdul Kadir, H. (2014). (R)-(+)-alpha-lipoic acid protected NG10815 cells against $\mathrm{H}(2) \mathrm{O}(2)$-induced cell death through PI3K-Akt/GSK-3beta pathway and suppression of NF-kappabeta-cytokines. Drug Des. Devel. Ther. 8, 1765-1780. doi: 10.2147/DDDT.S67980

Karunakaran, S., Diwakar, L., Saeed, U., Agarwal, V., Ramakrishnan, S., Iyengar, S., et al. (2007). Activation of apoptosis signal regulating kinase 1 (ASK1) and translocation of death-associated protein, Daxx, in substantia nigra pars compacta in a mouse model of Parkinson's disease: protection by alpha-lipoic acid. FASEB J. 21, 2226-2236. doi: 10.1096/fj.06-7580com

Kennedy, B. K., Berger, S. L., Brunet, A., Campisi, J., Cuervo, A. M., Epel, E. S., et al. (2014). Geroscience: linking aging to chronic disease. Cell 159, 709-713. doi: 10.1016/j.cell.2014.10.039

Kidd, P. M. (2005). Neurodegeneration from mitochondrial insufficiency: nutrients, stem cells, growth factors, and prospects for brain rebuilding using integrative management. Altern. Med. Rev. 10, 268-293. Available online at: http://www.altmedrev.com/publications/10/4/268.pdf

Kim, G. H., Kim, J. E., Rhie, S. J., and Yoon, S. (2015). The role of oxidative stress in neurodegenerative diseases. Exp. Neurobiol. 24, 325-340. doi: $10.5607 /$ en.2015.24.4.325

Koufaki, M. (2014). Therapeutic applications of lipoic acid: a patent review (2011-2014). Expert Opin. Ther. Pat. 24, 993-1005. doi: $10.1517 / 13543776.2014 .937425$

Kumaran, S., Panneerselvam, K. S., Shila, S., Sivarajan, K., and Panneerselvam, C. (2005). Age-associated deficit of mitochondrial oxidative phosphorylation in skeletal muscle: role of carnitine and lipoic acid. Mol. Cell Biochem. 280, 83-89. doi: 10.1007/s11010-005-8234-Z

Li, D. W., Li, G. R., Lu, Y., Liu, Z. Q., Chang, M., Yao, M., et al. (2013). alphalipoic acid protects dopaminergic neurons against MPP+-induced apoptosis by attenuating reactive oxygen species formation. Int J Mol Med 32, 108-114. doi: $10.3892 / \mathrm{ijmm} .2013 .1361$
Li, Y. H., He, Q., Yu, J. Z., Liu, C. Y., Feng, L., and Chai, Z., et al (2015). Lipoic acid protects dopaminergic neurons in LPS-induced Parkinson's disease model. Metab. Brain Dis. 30, 1217-1226. doi: 10.1007/s11011-015-9698-5

Limoli, C. L., Rola, R., Giedzinski, E., Mantha, S., Huang, T. T., and Fike, J. R. (2004). Cell-density-dependent regulation of neural precursor cell function. Proc. Natl. Acad. Sci. U.S.A. 101, 16052-16057. doi: 10.1073/pnas.0407065101

Lin, M. T., and Beal, M. F. (2006). Mitochondrial dysfunction and oxidative stress in neurodegenerative diseases. Nature 443, 787-795. doi: 10.1038/nature05292

Liu, J. (2008). The effects and mechanisms of mitochondrial nutrient $\alpha$-Lipoic acid on improving age-associated mitochondrial and cognitive dysfunction: an overview. Neurochem. Res. 33, 194-203. doi: 10.1007/s11064-007-9403-0

Liu, J., Head, E., Gharib, A. M., Yuan, W., Ingersoll, R. T., Hagen, T. M., et al. (2002a). Memory loss in old rats is associated with brain mitochondrial decay and RNA/DNA oxidation: partial reversal by feeding acetyl-L-carnitine and/or R-alpha -lipoic acid. Proc. Natl. Acad. Sci. U.S.A. 99, 2356-2361. doi: $10.1073 /$ pnas.261709299

Liu, J., Killilea, D. W., and Ames, B. N. (2002b). Age-associated mitochondrial oxidative decay: improvement of carnitine acetyltransferase substrate-binding affinity and activity in brain by feeding old rats acetyl-L- carnitine and/or R-alpha -lipoic acid. Proc. Natl. Acad. Sci. U.S.A. 99, 1876-1881. doi: $10.1073 /$ pnas.261709098

Liu, Z., Zhou, T., Ziegler, A. C., Dimitrion, P., and Zuo, L. (2017). Oxidative stress in neurodegenerative diseases: from molecular mechanisms to clinical applications. Oxid. Med. Cell Longev. 2017:2525967. doi: 10.1155/2017/2525967

Lodge, J. K., and Packer, L. (1999). "Natural sources of lipoic acid in plant and animal tissues," in Antioxidant Food Supplements in Human Health, eds L. Packer, M. Hiramatsu, and T. Yoshikawa (San Diego, CA: Academic Press), 121-134.

Lopez-Otin, C., Blasco, M. A., Partridge, L., Serrano, M., and Kroemer, G. (2013). The hallmarks of aging. Cell 153, 1194-1217. doi: 10.1016/j.cell.2013.05.039

Lovell, M. A., Xie, C., Xiong, S., and Markesbery, W. R. (2003). Protection against amyloid beta peptide and iron/hydrogen peroxide toxicity by alpha lipoic acid. J. Alzheimers Dis. 5, 229-239. doi: 10.3233/JAD-2003-5306

Ma, R., Wang, X., Peng, P., Xiong, J., Dong, H., Wang, L., et al. (2016). alphaLipoic acid inhibits sevoflurane-induced neuronal apoptosis through PI3K/Akt signalling pathway. Cell Biochem. Funct. 34, 42-47. doi: 10.1002/cbf.3163

Mahboob, A., Farhat, S. M., Iqbal, G., Babar, M. M., Zaidi, N. U., Nabavi, S. M., et al. (2016). Alpha-lipoic acid-mediated activation of muscarinic receptors improves hippocampus- and amygdala-dependent memory. Brain Res. Bull. 122, 19-28. doi: 10.1016/j.brainresbull.2016.02.014

Manda, K., Ueno, M., Moritake, T., and Anzai, K. (2007). Radiation-induced cognitive dysfunction and cerebellar oxidative stress in mice: protective effect of alpha-lipoic acid. Behav. Brain Res. 177, 7-14. doi: 10.1016/j.bbr.2006.11.013

Mehrotra, A., Kanwal, A., Banerjee, S. K., and Sandhir, R. (2015). Mitochondrial modulators in experimental Huntington's disease: reversal of mitochondrial dysfunctions and cognitive deficits. Neurobiol. Aging 36, 2186-2200. doi: 10.1016/j.neurobiolaging.2015.02.004

Militao, G. C., Ferreira, P. M., and de Freitas, R. M. (2010). Effects of lipoic acid on oxidative stress in rat striatum after pilocarpine-induced seizures. Neurochem. Int. 56, 16-20. doi: 10.1016/j.neuint.2009.08.009

Miquel, J. (2002). Can antioxidant diet supplementation protect against age-related mitochondrial damage? Ann. N.Y. Acad. Sci. 959, 508-516. doi: 10.1111/j.1749-6632.2002.tb02120.x

Moini, H., Packer, L., and Saris, N. E. (2002). Antioxidant and prooxidant activities of alpha-lipoic acid and dihydrolipoic acid. Toxicol. Appl. Pharmacol. 182, 84-90. doi: 10.1006/taap.2002.9437

Moreira, P. I., Harris, P. L., Zhu, X., Santos, M. S., Oliveira, C. R., Smith, M. A., et al. (2007). Lipoic acid and $\mathrm{N}$-acetyl cysteine decrease mitochondrial-related oxidative stress in Alzheimer disease patient fibroblasts. J. Alzheimers Dis. 12, 195-206. doi: 10.3233/JAD-2007-12210

Morikawa, T., Yasuno, R., and Wada, H. (2001). Do mammalian cells synthesize lipoic acid? Identification of a mouse cDNA encoding a lipoic acid synthase located in mitochondria. FEBS Lett. 498, 16-21. doi: 10.1016/S0014-5793(01)02469-3

Ono, K., Hirohata, M., and Yamada, M. (2006). Alpha-lipoic acid exhibits antiamyloidogenicity for beta-amyloid fibrils in vitro. Biochem. Biophys. Res. Commun. 341, 1046-1052. doi: 10.1016/j.bbrc.2006.01.063 
Packer, L., and Cadenas, E. (2011). Lipoic acid: energy metabolism and redox regulation of transcription and cell signaling. J. Clin. Biochem. Nutr. 48, 26-32. doi: 10.3164/jcbn.11-005FR

Packer, L., Kraemer, K., and Rimbach, G. (2001). Molecular aspects of lipoic acid in the prevention of diabetes complications. Nutrition 17, 888-895. doi: 10.1016/S0899-9007(01)00658-X

Packer, L., Tritschler, H. J., and Wessel, K. (1997). Neuroprotection by the metabolic antioxidant alpha-lipoic acid. Free Radic. Biol. Med. 22, 359-378. doi: 10.1016/S0891-5849(96)00269-9

Packer, L., Witt, E. H., and Tritschler, H. J. (1995). alpha-Lipoic acid as a biological antioxidant. Free Radic. Biol. Med. 19, 227-250. doi: 10.1016/0891-5849(95)00017-R

Palaniappan, A. R., and Dai, A. (2007). Mitochondrial ageing and the beneficial role of alpha-lipoic acid. Neurochem. Res. 32, 1552-1558. doi: 10.1007/s11064-007-9355-4

Park, S., Karunakaran, U., Jeoung, N. H., Jeon, J. H., and Lee, I. K. (2014). Physiological effect and therapeutic application of alpha lipoic acid. Curr. Med. Chem. 21, 3636-3645. doi: 10.2174/0929867321666140706141806

Payton, A., Gibbons, L., Davidson, Y., Ollier, W., Rabbitt, P., Worthington, J., et al. (2005). Influence of serotonin transporter gene polymorphisms on cognitive decline and cognitive abilities in a nondemented elderly population. Mol. Psychiatry 10, 1133-1139. doi: 10.1038/sj.mp.4001733

Pizza, V., Agresta, A., D’Acunto, C. W., Festa, M., and Capasso, A. (2011). Neuroinflammation and ageing: current theories and an overview of the data. Rev. Recent Clin. Trials 6, 189-203. doi: 10.2174/157488711796575577

Poon, H. F., Farr, S. A., Thongboonkerd, V., Lynn, B. C., Banks, W. A., Morley, J. E., et al. (2005). Proteomic analysis of specific brain proteins in aged SAMP8 mice treated with alpha-lipoic acid: implications for aging and age-related neurodegenerative disorders. Neurochem. Int. 46, 159-168. doi: 10.1016/j.neuint.2004.07.008

Quinn, J. F., Bussiere, J. R., Hammond, R. S., Montine, T. J., Henson, E., Jones, R. E., et al. (2007). Chronic dietary alpha-lipoic acid reduces deficits in hippocampal memory of aged Tg2576 mice. Neurobiol. Aging 28, 213-225. doi: 10.1016/j.neurobiolaging.2005.12.014

Reed, L. J., De, B. B., Gunsalus, I. C., and Hornberger, C. S. Jr. (1951). Crystalline alpha-lipoic acid; a catalytic agent associated with pyruvate dehydrogenase. Science 114, 93-94. doi: 10.1126/science.114.2952.93

Rochette, L., Ghibu, S., Richard, C., Zeller, M., Cottin, Y., and Vergely, C. (2013). Direct and indirect antioxidant properties of alpha-lipoic acid and therapeutic potential. Mol. Nutr. Food Res. 57, 114-125. doi: 10.1002/mnfr.201200608

Ross, C. A., Aylward, E. H., Wild, E. J., Langbehn, D. R., Long, J. D., Warner, J. H., et al. (2014). Huntington disease: natural history, biomarkers and prospects for therapeutics. Nat. Rev. Neurol. 10, 204-216. doi: 10.1038/nrneurol.2014.24

Sancheti, H., Akopian, G., Yin, F., Brinton, R. D., Walsh, J. P., and Cadenas, E. (2013). Age-dependent modulation of synaptic plasticity and insulin mimetic effect of lipoic acid on a mouse model of Alzheimer's disease. PLoS ONE 8:e69830. doi: 10.1371/journal.pone.0069830

Santos, R. X., Correia, S. C., Zhu, X., Smith, M. A., Moreira, P. I., Castellani, R. J., et al. (2013). Mitochondrial DNA oxidative damage and repair in aging and Alzheimer's disease. Antioxid Redox Signal 18, 2444-2457. doi: 10.1089/ars.2012.5039

Savitha, S., and Panneerselvam, C. (2006). Mitochondrial membrane damage during aging process in rat heart: potential efficacy of L-carnitine and DL alpha lipoic acid. Mech. Ageing Dev. 127, 349-355. doi: 10.1016/j.mad.2005.12.004

Seidman, M. D., Khan, M. J., Bai, U., Shirwany, N., and Quirk, W. S. (2000). Biologic activity of mitochondrial metabolites on aging and age-related hearing loss. Am. J. Otol. 21, 161-167. doi: 10.1016/S0196-0709(00)80003-4

Shay, K. P., Moreau, R. F., Smith, E. J., Smith, A. R., and Hagen, T. M. (2009). Alpha-lipoic acid as a dietary supplement: molecular mechanisms and therapeutic potential. Biochim. Biophys. Acta 1790, 1149-1160. doi: 10.1016/j.bbagen.2009.07.026

Siedlak, S. L., Casadesus, G., Webber, K. M., Pappolla, M. A., Atwood, C. S., Smith, M. A., et al. (2009). Chronic antioxidant therapy reduces oxidative stress in a mouse model of Alzheimer's disease. Free Radic. Res. 43, 156-164. doi: 10.1080/10715760802644694

Singh, G., Pachouri, U., Khaidem, D., Kundu, A., Chopra, C., and Singh, P. (2015). Mitochondrial DNA Damage and Diseases [Version 1; Referees: 1 Approved].

Snell, E. E., Strong, F. M., and Peterson, W. H. (1937). Growth factors for bacteria: fractionation and properties of an accessory factor for lactic acid bacteria. Biochem. J. 31, 1789-1799. doi: 10.1042/bj0311789

Stoll, S., Hartmann, H., Cohen, S. A., and Muller, W. E. (1993). The potent free radical scavenger alpha-lipoic acid improves memory in aged mice: putative relationship to NMDA receptor deficits. Pharmacol. Biochem. Behav. 46, 799-805. doi: 10.1016/0091-3057(93)90204-7

Stoll, S., Rostock, A., Bartsch, R., Korn, E., Meichelbock, A., and Muller, W. E. (1994). The potent free radical scavenger alpha-lipoic acid improves cognition in rodents. Ann. N.Y. Acad. Sci. 717, 122-128. doi: 10.1111/j.1749-6632.1994.tb12079.x

Suh, J. H., Moreau, R., Heath, S. H., and Hagen, T. M. (2005). Dietary supplementation with (R)-alpha-lipoic acid reverses the age-related accumulation of iron and depletion of antioxidants in the rat cerebral cortex. Redox Rep. 10, 52-60. doi: 10.1179/135100005X21624

Thakurta, I. G., Banerjee, P., Bagh, M. B., Ghosh, A., Sahoo, A., Chattopadhyay, S., et al. (2014). Combination of $\mathrm{N}$-acetylcysteine, alpha-lipoic acid and alpha-tocopherol substantially prevents the brain synaptosomal alterations and memory and learning deficits of aged rats. Exp. Gerontol. 50, 19-25. doi: 10.1016/j.exger.2013.11.008

Tirosh, O., Sen, C. K., Roy, S., Kobayashi, M. S., and Packer, L. (1999). Neuroprotective effects of alpha-lipoic acid and its positively charged amide analogue. Free Radic. Biol. Med. 26, 1418-1426. doi: 10.1016/S0891-5849(99)00014-3

Virmani, A., Pinto, L., Binienda, Z., and Ali, S. (2013). Food, nutrigenomics, and neurodegeneration-neuroprotection by what you eat! Mol. Neurobiol. 48, 353-362. doi: 10.1007/s12035-013-8498-3

Xing, Z. G., Yu, G. D., Qin, L., Jiang, F., and Zhao, W. H. (2015). Effects and mechanism of lipoic acid on beta-amyloid-intoxicated C6 glioma cells. Genet Mol. Res. 14, 13880-13888. doi: 10.4238/2015.October.29.8

Yamada, T., Hashida, K., Takarada-Iemata, M., Matsugo, S., and Hori, O. (2011). alpha-Lipoic acid (LA) enantiomers protect SH-SY5Y cells against glutathione depletion. Neurochem. Int. 59, 1003-1009. doi: 10.1016/j.neuint.2011.09.005

Zaitone, S. A., Abo-Elmatty, D. M., and Shaalan, A. A. (2012). Acetyl-L-carnitine and alpha-lipoic acid affect rotenone-induced damage in nigral dopaminergic neurons of rat brain, implication for Parkinson's disease therapy. Pharmacol. Biochem. Behav. 100, 347-360. doi: 10.1016/j.pbb.2011.09.002

Zhang, H., Jia, H., Liu, J., Ao, N., Yan, B., Shen, W., et al. (2010). Combined R-alpha-lipoic acid and acetyl-L-carnitine exerts efficient preventative effects in a cellular model of Parkinson's disease. J. Cell Mol. Med. 14, 215-225. doi: 10.1111/j.1582-4934.2008.00390.x

Zhang, L., Xing, G. Q., Barker, J. L., Chang, Y., Maric, D., Ma, W., et al. (2001). Alpha-lipoic acid protects rat cortical neurons against cell death induced by amyloid and hydrogen peroxide through the Akt signalling pathway. Neurosci. Lett. 312, 125-128. doi: 10.1016/S0304-3940(01)02205-4

Zuo, L., and Motherwell, M. S. (2013). The impact of reactive oxygen species and genetic mitochondrial mutations in Parkinson's disease. Gene 532, 18-23. doi: 10.1016/j.gene.2013.07.085

Conflict of Interest Statement: The authors declare that the research was conducted in the absence of any commercial or financial relationships that could be construed as a potential conflict of interest.

Copyright (c) 2017 Molz and Schröder. This is an open-access article distributed under the terms of the Creative Commons Attribution License (CC BY). The use, distribution or reproduction in other forums is permitted, provided the original author(s) or licensor are credited and that the original publication in this journal is cited, in accordance with accepted academic practice. No use, distribution or reproduction is permitted which does not comply with these terms. 